\title{
Non-invasive, energy-based assessment of patient-specific material properties of arterial tissue
}

\author{
M. Smoljkić • J. Vander Sloten • \\ P. Segers • N. Famaey
}

Received: date / Accepted: date

\begin{abstract}
The mechanical properties of human biological tissue vary greatly. The determination of arterial material properties should be based on experimental data, i.e. diameter, length, intramural pressure, axial force and stress-free geometry. Currently, clinical data provide only non-invasively measured pressurediameter data for superficial arteries (e.g. common carotid and femoral artery). The lack of information forces us to take into account certain assumptions regarding the in situ configuration to estimate material properties in vivo. This paper proposes a new, non-invasive, energy-based approach for arterial material property estimation. This approach is compared with an approach proposed in literature. For this purpose, a simplified finite element model of an artery was used as a mock experimental situation. This method enables exact knowledge of the actual material properties, thereby allowing a quantitative evaluation of material property estimation approaches. The results show that imposing conditions on strain energy can provide a good estimation of the material properties from the non-invasively measured pressure and diameter data.
\end{abstract}

Keywords Arteries - Material properties · Constitutive models $\cdot$ Parameter estimation

\footnotetext{
M. Smoljkić · J. Vander Sloten · N. Famaey

Biomechanics Section,

Faculty of Mechanical Engineering, KU Leuven

Celestijnenlaan 300, b2419

Leuven, Belgium

Tel.: +32 16372824

E-mail: marija.smoljkic@kuleuven.be

P. Segers

IBiTech-bioMMeda, iMinds Medical IT,

Ghent University, Gent, Belgium
}

\section{Introduction}

Cardiovascular diseases are the leading cause of death in Europe, claiming $47 \%$ of all deaths per year (Nichols et al., 2013). For pathologies such as artery stenosis, aortic aneurysms, valve repair or replacement a minimally invasive procedure has become the preferred method of treatment. Pre-operative, patient-specific simulations of these procedures in complex cases can offer valuable information to a surgeon, as a training tool, for outcome prediction and device sizing. These simulations should optimally reflect the patient specific mechanical behaviour of the cardiovascular tissue.

To describe the nonlinear, anisotropic behaviour of arteries, which undergo large deformations, a large number of constitutive models have been developed (Vito and Dixon, 2003; Gasser et al., 2006). Identification of material parameters for these models requires a substantial amount of experimental data from loading experiments in different directions. These are classically obtained through in vitro biaxial testing, and are then fitted to the constitutive equation. For clinical applications, where the goal is to use patient-specific parameters, this approach cannot be applied due to the necessity to dissect the specimen. This makes the transition from invasive in vitro to non-invasive in vivo parameter identification a crucial step. Non-invasive estimation of material properties, however, still remains a challenging task.

In this paper, the focus lies on defining the material properties of arteries through non-invasively measured pressure and diameter data. In the classical, invasive or ex vivo approach, inflation-extension tests are performed, and the circular segment is pressurized while the change in diameter and axial force are measured. Prior to the pressurization, the segment is stretched to 
its physiological axial prestretch. In the in vivo situation all the axial information, i.e. the prestretch and the axial force, are unknown. The lack of knowledge about the magnitude of the circumferential residual stresses present in the arteries (Cardamone et al., 2009; Rachev and Greenwald, 2003) further complicates the parameter estimation.

To the authors' knowledge, the first attempt to overcome the lack of axial force information in the process of estimating the parameters of advanced constitutive models was proposed in Schulze-Bauer and Holzapfel (2003). They assumed that the axial prestretch and force remain constant in the physiological pressure range - a concept first suggested in Weizsäcker et al. (1983) and that "the ratio of circumferential to axial stress is known for one (arbitrary) internal pressure". The used value for this ratio was derived from experimental data for rabbit thoracic aortas (Fung et al., 1979). They applied their approach on a Fung-type strain energy density function (SEDF) which takes into account the typical nonlinear and anisotropic response. This approach was later adopted by Stålhand (2009) on a twofibre family Holzapfel-Gasser-Ogden model proposed in Holzapfel et al. (2000). Both of these approaches only take into account the passive behaviour of the arterial segment. In Masson et al. (2008) non-invasively obtained pressure-diameter data was fitted, without additional conditions, for the human common carotid artery with a four-fibre family model, thereby adding the active contribution of the smooth muscle cells. Including smooth muscle cells results in additional parameters which are unknown a priori and need to be obtained from a parameter estimation procedure. This can result in over-parameterisation. A short overview of all work done so far in the field of non-invasive parameter estimation for arteries can be found in van der Horst et al. (2012). What is common to all the methods is that they suffer the lack of proper validation, making it difficult to assess their value.

The first contribution of this paper is a finite element (FE) based method to quantitatively validate the fitting approaches proposed in literature. The second contribution is the development of a new and improved approach for non-invasive parameter estimation.

In the following sections, the material model is introduced, the FE based validation method is described and the parameter identification procedure is explained. Next, the results of the different approaches are presented, followed by a discussion and a description of future work.

\section{Material and Methods}

\subsection{Kinematics}

If the artery is considered to be a thick-walled cylinder, and any material point on the cylinder is defined by its cylindrical coordinates, the deformation during the cardiac cycle can be described by a deformation gradient. Depending on the different loading states, three configurations can be defined as shown on Figure 1 . In the $\mathcal{R}_{0}$

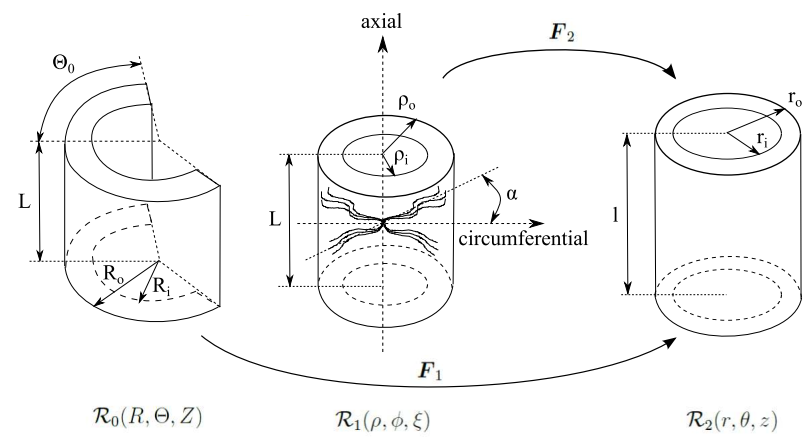

Fig. 1 Stress free $\left(\mathcal{R}_{0}\right)$, unloaded $\left(\mathcal{R}_{1}\right)$ and loaded $\left(\mathcal{R}_{2}\right)$ configuration

configuration there are no radial, circumferential or axial stresses and the artery is an open segment. Accordingly, this configuration is named the stress-free configuration. $\mathcal{R}_{1}$, i.e. the unloaded configuration, represents the state where the residual, circumferential stresses are present but the intraluminal pressure is zero and there is no axial prestretch. Finally, the $\mathcal{R}_{2}$ configuration describes the loading state of the artery with pressure and a certain axial stretch applied to the segment. Depending on which of the configurations is chosen as a reference, the mapping is described with the corresponding deformation gradient. In this paper, both $\mathcal{R}_{0}$ and $\mathcal{R}_{1}$ will be used as the reference configuration, so deformation gradients $\boldsymbol{F}_{1}$ and $\boldsymbol{F}_{2}$ need to be defined (see Figure 1) (Humphrey, 2002):

$$
\begin{aligned}
& \boldsymbol{F}_{1}=\left[\begin{array}{ccc}
\frac{\partial r}{\partial R} & 0 & 0 \\
0 & \frac{\pi r}{\Theta_{0} \mathrm{R}} & 0 \\
0 & 0 & \Lambda \lambda
\end{array}\right]=\operatorname{diag}\left(\lambda_{r} \lambda_{\theta} \lambda_{z}\right), \\
& \boldsymbol{F}_{2}=\left[\begin{array}{ccc}
\frac{\partial r}{\partial \rho} & 0 & 0 \\
0 & \frac{r}{\rho} & 0 \\
0 & 0 & \lambda
\end{array}\right]=\operatorname{diag}\left(\lambda_{r} \lambda_{\theta} \lambda_{z}\right),
\end{aligned}
$$

where $R_{i} \leq R \leq R_{o}, \rho_{i} \leq \rho \leq \rho_{o}$, and $r_{i} \leq r \leq r_{o}$ denote the radii in different configurations, and the subscripts $i$ and $o$ denote the radius at the inner and outer wall. $\Theta_{0}$ is the opening angle in the stress-free configuration (Figure 1). $\Lambda$ is the axial stretch ratio between the stress-free $\left(\mathcal{R}_{0}\right)$ and unloaded $\left(\mathcal{R}_{1}\right)$ configuration 
which is reported to be near unity (Humphrey, 2002). Hence, in this paper, $\Lambda$ is set to 1 . The ratio of the length in the loaded configuration $(l)$ and the length in the unloaded configuration $(L)$ is marked with $\lambda$.

\subsection{Material model}

This study uses the material model proposed in Gasser et al. (2006), further on referred to as the HGO model. The model represents an artery as an incompressible, fibre-reinforced structure, taking into account the collagen fibre dispersion. The $\operatorname{SEDF}(\Psi)$ consists of an isotropic and an anisotropic part, the former representing the contribution of the matrix material ( $\left.\Psi_{\text {mat }}\right)$ and the latter the contribution of the collagen fibre families embedded in the matrix material $\left(\Psi_{\text {coll }}\right)$ :

$\Psi=\Psi_{\text {mat }}+\Psi_{\text {coll }}$.

The contribution of the matrix material is described with a neo-Hookean model:

$\Psi_{\text {mat }}=\frac{\mu}{2}\left(I_{1}-3\right), \quad I_{1}=\lambda_{r}^{2}+\lambda_{\theta}^{2}+\lambda_{z}^{2}$,

where $\mu>0$ is a stress-like parameter which represents the stiffness of the matrix material. The first invariant of the right Cauchy-Green strain tensor, marked with $I_{1}$, is written as a function of the three principal stretches $\lambda_{i}, i=r, \theta, z$, in the radial, circumferential, and axial directions of the vessel, respectively. From the incompressibility condition the relation between the stretches can be expressed as:

$\lambda_{r} \lambda_{\theta} \lambda_{z}=1$

The strain energy stored in the collagen fibres is described with an exponential function which incorporates the stiffening effect that occurs at higher pressures:

$$
\begin{gathered}
\Psi_{\mathrm{coll}}=\frac{k_{1}}{k_{2}}\left\{\exp \left[k_{2}\left(\kappa I_{1}+(1-3 \kappa) I_{4}-1\right)^{2}\right]-1\right\}, \\
I_{4}=\lambda_{z}^{2} \sin ^{2} \alpha+\lambda_{\theta}^{2} \cos ^{2} \alpha .
\end{gathered}
$$

$k_{1}>0$ represents the stiffness of the collagen fibres and it is, like $\mu$, a stress-like parameter. $k_{2}$ is a dimensionless parameter, greater than zero, which contributes to the stiffening of the arterial response with increasing loading pressure. The artery is assumed to consist of two fibre families which are embedded into the matrix material and are oriented symmetrically with respect to the axial direction. $\kappa \in[0,1 / 3]$ describes the dispersion of the collagen fibres. The two fibre families have the same material properties. $I_{4}$ is the invariant related to the orientation of the collagen fibre families defined by the angle $\alpha$, i.e. the angle between the circumferential and axial direction (see Figure 1).

To summarize, there are three material parameters $\mu, k_{1}, k_{2}$ and two structural parameters $\alpha$ and $\kappa$ that need to be determined through a parameter identification procedure as described in section 2.4.

\subsection{Finite element model}

To evaluate different parameter fitting approaches a FE model of an aorta was built in Abaqus/Standard version 6.10-2. The artery was modelled as a cylindrical tube with the HGO model as described in section 2.2. $\mathrm{C} 3 \mathrm{D} 8 \mathrm{H}$ elements were assigned to the mesh. The assigned material properties were acquired from the raw pressure-diameter data obtained from experiments on rat abdominal aortas performed in our research group as described in Famaey et al. (2012) ( $\mu=15 \mathrm{kPa}$, $k_{1}=80 \mathrm{kPa}, k_{2}=5, \alpha=5^{\circ}$, and $\left.\kappa=0.2\right)$. The dimensions of the modelled aorta were also based on rat abdominal aorta dimensions. The model starts from an initial stress-free geometry (Figure 2a) with an angle $\Theta_{0}=120^{\circ}$, wall thickness $H=0.14 \mathrm{~mm}$, length $L=1 \mathrm{~mm}$, and $R_{0}=1.14 \mathrm{~mm}$ (Famaey et al., 2012). Since the aorta was considered to be a perfectly cylindrical segment, only half of the cylinder was modelled. The loading was split in different steps, as schemat-

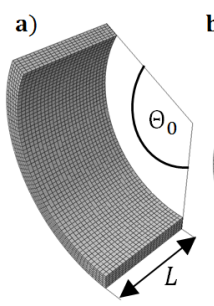

b)

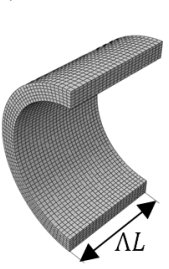

c)

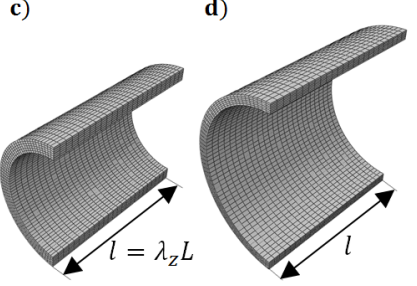

Fig. 2 Different loading steps: a) initial stress-free configuration, $\mathcal{R}_{0} ;$ b) closed segment (unloaded configuration, $\mathcal{R}_{1}$ ); c) stretched segment; d) loaded configuration (pressurization), $\mathcal{R}_{2}$.

ically shown in Figure 2. First, the open segment is closed to form a cylinder. This is followed by applying the axial prestretch and pressurizing the segment. The applied pressures ranged from $9.33 \mathrm{kPa}(70 \mathrm{mmHg})$ to $16 \mathrm{kPa}(120 \mathrm{mmHg})$, which are values corresponding to diastolic and systolic levels of healthy rats and humans alike.

The axial force exerted on the arterial tissue, also known as the reduced axial force, is reported to be approximately constant in the physiological pressure range, from diastole to systole (Ogden, 2009). To en- 
sure that this is the case in our simulation we prescribed different axial prestrains from $10 \%$ to $100 \%$. The corresponding reduced axial forces are shown in Figure 3 . The axial prestretch $\lambda_{z}=1.6$ was chosen, as this yields an approximately constant reduced axial force. This value was also close to the values experimentally observed for rat abdominal aorta (Famaey et al., 2012). From the FE simulation, the reduced axial force

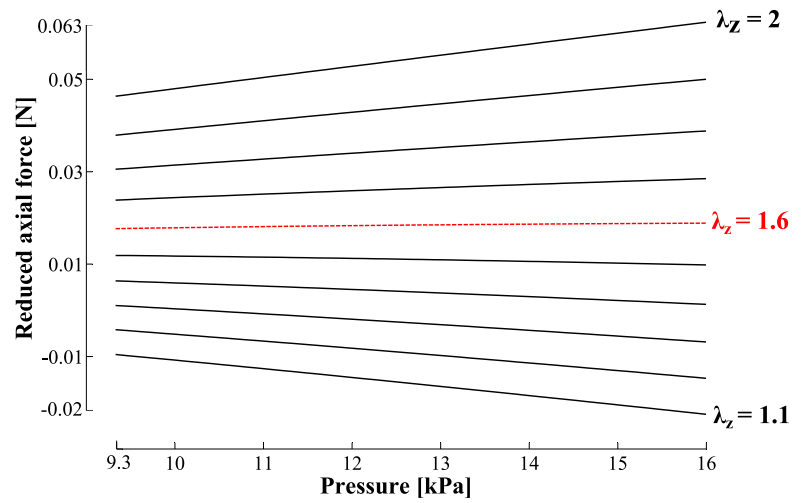

Fig. 3 Reduced axial force for different values of axial stretch $\left(\lambda_{z}\right)$. The force remains approximately constant for $\lambda_{z}=1.6$.

$\left(\boldsymbol{F}^{F E M}\right)$ and the outer radius $\left(\boldsymbol{r}_{o}\right)$ were extracted. Together with the applied pressure $\left(\boldsymbol{P}^{F E M}\right)$, these values were used as a substitute for the experimentally measured ones.

\subsection{Parameter identification methods}

For all methods presented below, the general approach is to minimize the difference between the measured ( $i n$ casu simulated) values and the same values predicted by the constitutive model in an iterative process. The parameters were fitted using Matlab R2010b (The MathWorks, Natick, MA, USA), with the lsqnonlin routine and the trust-region-reflective optimization algorithm. Ten initial parameter sets were given as initial points (Multistart function), distributed over the entire fitting range. To have more equally distributed search areas, the parameters were scaled, as well as their corresponding fitting range. The fitting range, as well as the corresponding scaling factor, for each parameter and for each method is shown in Table 1. Parameter $\alpha$ was fitted in radians. Note that Table 1 reports unscaled fitting ranges and $\alpha$ in degrees.

Two scenarios were compared, each with their proper objective function. In the first, so-called 'invasive approach' is assumed that in vitro experiments were conducted and that pressure, diameter and axial force are known from the measurements. The second scenario, namely the 'non-invasive approach', assumes that the axial force is unknown.

In the following section these scenarios are explained. For each scenario two approaches are given. The minimized objective function for each approach is stated, followed by an explanation of its constituents.

\subsubsection{Invasive approaches}

The minimized objective function in both invasive approaches is:

$$
\begin{aligned}
\varepsilon(\text { pars })= & \sum_{j=1}^{n}\left\{\left[w_{p}\left(P_{j}^{\text {mod }}-P_{j}^{F E M}\right)\right]^{2}\right. \\
& \left.+\left[w_{f}\left(\frac{F_{j}^{\text {mod }}}{A_{j}^{\text {mod }}}-\frac{F_{j}^{F E M}}{A_{j}^{\text {mod }}}\right)\right]^{2}\right\},
\end{aligned}
$$

where $n$ is the total number of data points considered and $j$ denotes a certain data record corresponding to a certain pressure level. pars represents a vector of fitted parameters. In both of the invasive approaches the only unknown parameters are the ones from the material model thus pars $=\left(c, k_{1}, k_{2}, \alpha, \kappa\right)$. The pressures and reduced axial forces predicted by the constitutive model are marked with $P_{j}^{\text {mod }}$ and $F_{j}^{\text {mod }}$. The corresponding values representing the experimental measurements (in this case simulated using the FE model) are $P_{j}^{F E M}$ and $F_{j}^{F E M} . A_{j}^{m o d}$ is the current cross sectional area calculated as:

$A_{j}^{\text {mod }}=\pi\left[\left(R_{o} \lambda_{o, j}\right)^{2}-\left(R_{i} \lambda_{i, j}\right)^{2}\right]$.

$R_{o}$ and $R_{i}$ are the outer and inner radius, in the reference configuration, respectively. $\lambda_{i, j}$ and $\lambda_{o, j}$ represent the circumferential stretches at the inner and outer wall for every data record. The weighting factors in (6) will depend on the used units and in our case were set to 1 for $w_{p}$ and $10^{2}$ for $w_{f}$.

Invasive approach without residual stresses (Inv noRS) The internal pressures $\left(\boldsymbol{P}^{\text {mod }}\right)$ and reduced axial forces $\left(\boldsymbol{F}^{\text {mod }}\right)$ can be derived as a function of $\lambda_{\theta}$ and $\lambda_{z}$. A detailed derivation can be found in Ogden (2009). As mentioned earlier, two approaches are possible. In the first, the circumferential residual stress, related to the opening angle, is not taken into account and the unloaded configuration $\left(\mathcal{R}_{1}\right)$ is considered to be the reference configuration. In this case, the final equations are:

$P_{j}^{\text {mod }}=\int_{\lambda_{\mathrm{o}}}^{\lambda_{\mathrm{i}}}\left(\lambda_{\theta}^{2} \lambda_{z}-1\right)^{-1} \frac{\partial \Psi}{\partial \lambda_{\theta}} d \lambda_{\theta}$, 


$$
\begin{aligned}
F_{j}^{\text {mod }}= & \pi \rho_{\mathrm{i}}^{2}\left(\lambda_{\theta, \mathrm{i}}^{2} \lambda_{z}-1\right) \int_{\lambda_{\mathrm{o}}}^{\lambda_{\mathrm{i}}}\left(\lambda_{\theta}^{2} \lambda_{z}-1\right)^{-2} \\
& \left(2 \lambda_{z} \frac{\partial \Psi}{\partial \lambda_{z}}-\lambda \frac{\partial \Psi}{\partial \lambda_{\theta}}\right) \lambda_{\theta} d \lambda_{\theta}
\end{aligned}
$$

where $\lambda_{\mathrm{i}}$ and $\lambda_{\mathrm{o}}$ are the circumferential stretches at the inner and the outer wall, respectively. $\Psi$ is the SEDF as, in our case, described in (2). The circumferential stretches are calculated as $\lambda_{\theta}=r / \rho$. $\Lambda$, see Figure 2, is considered to be 1 so the axial stretches are $\lambda_{z}=l / L$. The axial stretches can also be derived by assuming incompressibility:

$\lambda_{z}=\frac{\rho_{\mathrm{o}}^{2}-\rho_{\mathrm{i}}^{2}}{r_{\mathrm{o}}^{2}-r_{\mathrm{i}}^{2}}$.

Invasive approach with residual stresses (Inv $R S$ ) In the second approach, the circumferential residual stresses are incorporated by means of an opening angle. Then the equations for internal pressures and reduced axial force calculations are:

$$
P_{j}^{\text {mod }}=\frac{\pi}{\Theta_{0}} \int_{\lambda_{\mathrm{o}}}^{\lambda_{\mathrm{i}}}\left(\lambda_{\theta}^{2} \lambda_{z}-\frac{\pi}{\Theta_{0}}\right)^{-1} \frac{\partial \Psi}{\partial \lambda_{\theta}} d \lambda_{\theta}
$$

$$
\begin{aligned}
F_{j}^{\text {mod }}= & \Theta_{0} R_{\mathrm{i}}^{2}\left(\lambda_{\theta, \mathrm{i}}^{2} \lambda_{z}-\frac{\pi}{\Theta_{0}}\right) \int_{\lambda_{\circ}}^{\lambda_{\mathrm{i}}}\left(\lambda_{\theta}^{2} \lambda_{z}-\frac{\pi}{\Theta_{0}}\right)^{-2} \\
& \left(2 \lambda_{z} \frac{\partial \Psi}{\partial \lambda_{z}}-\lambda \frac{\partial \Psi}{\partial \lambda_{\theta}}\right) \lambda_{\theta} d \lambda_{\theta}
\end{aligned}
$$

Denotation remains the same as for equations (8) and (9). The equations for circumferential and axial stretches need to be changed accordingly. The circumferential stretches are now calculated as $\lambda_{\theta}=r \pi / R \Theta_{0}$, and axial stretches are again $\lambda_{z}=l / L$. Incorporating the incompressibility condition gives us the following equation for the axial stretch:

$\lambda_{z}=\frac{\Theta_{0}\left(R_{\mathrm{o}}^{2}-R_{\mathrm{i}}^{2}\right)}{\pi\left(r_{\mathrm{o}}^{2}-r_{\mathrm{i}}^{2}\right)}$.

\subsubsection{Non-invasive approaches}

This section first repeats an existing method proposed by Schulze-Bauer and Holzapfel (2003) and adopted in Stålhand (2009), further on referred to as the noninvasive approach I, and then proposes a new and improved method, further on referred as non-invasive approach II.
Non-invasive approach I (NonInv I) Analogous to the invasive approach, an objective function needs to be minimized. For NonInv I, this objective function is:

$\varepsilon($ pars $)=\sum_{j=1}^{n}\left[\left(\sigma_{\theta \theta, j}^{\bmod }-\sigma_{\theta \theta, j}\right)^{2}+\left(\sigma_{z z, j}^{\bmod }-\sigma_{z z, j}\right)^{2}\right]$.

$\sigma_{\theta \theta, j}^{\bmod }$ and $\sigma_{z z, j}^{\bmod }$ are the circumferential and axial stresses predicted by the constitutive model for the $j$-th data record. They are derived as follows (Schulze-Bauer and Holzapfel, 2003):

$\sigma_{\theta \theta, j}^{m o d}=\lambda_{\theta} \frac{\partial \Psi}{\partial \lambda_{\theta}}, \quad \sigma_{z z, j}^{m o d}=\lambda_{z} \frac{\partial \Psi}{\partial \lambda_{z}}$.

In this approach, the artery is considered to be a thin walled tube. Hence, the circumferential stress $\sigma_{\theta \theta, j}$ in (14) can be calculated from Laplace's law (16). The axial stress can be calculated by enforcing the equilibrium and assuming that the axial force is constant (17):

$\sigma_{\theta \theta, j}=\frac{P_{j}^{F E M} d_{\mathrm{i}, j}}{2 h_{j}}$,

$\sigma_{z z, j}=\frac{d_{\mathrm{i}, j}^{2} P_{j}^{F E M} \pi+4 F^{e s t}}{4 h_{j}\left(d_{\mathrm{i}, j}+h_{j}\right) \pi}$.

Here, $h_{j}$ represents the current wall thickness, $d_{\mathrm{i}, j}$ current inner diameter and $F^{e s t}$ is the estimated reduced axial force. As it is impossible to obtain the latter from noninvasive measurements, following Schulze-Bauer and Holzapfel (2003), it is assumed that the ratio of principal stresses $\gamma=\sigma_{z z} / \sigma_{\theta \theta}$ is known for a particular pressure $\bar{P}$. Once $\gamma$ is determined, $F^{e s t}$ can be calculated as:

$F^{e s t}=\frac{1}{4} \bar{d}_{\mathrm{i}} \bar{P} \pi\left(2 \bar{d}_{\mathrm{i}} \gamma-\bar{d}_{\mathrm{i}}+2 \bar{h} \gamma\right)$,

where $\bar{d}_{\mathrm{i}}$ is the inner diameter and $\bar{h}$ is the wall thickness associated with $\bar{P}$. The estimated $F^{e s t}$ is assumed to be constant for all pressures in the physiological range. For a more detailed explanation of equations 14 to 18 , the interested reader is referred to Schulze-Bauer and Holzapfel (2003).

To evaluate the sensitivity to this value, different values for $\gamma$ were used, ranging from 0.1 to 1 . Figure 6 shows the dependence of the root mean square error on the parameter $\gamma$. Specific attention was paid to two values of $\gamma$ : The first value, $\gamma=0.8565$, is the correct ratio acquired from the FE simulation, e.g. at pressure $\bar{P}=13.3 \mathrm{kPa}(100 \mathrm{mmHg})$. The parameters obtained using this value are reported in Table 1 under the name NonInv Ia. The second value is the one that was actually used in Schulze-Bauer and Holzapfel (2003) and Stålhand (2009), $\gamma$ being 0.59 at $\bar{P}=13.3$ $\mathrm{kPa}$, a value derived from literature (Fung et al., 1979). 
For this case, the parameters are reported under the name NonInv $I b$ in Table 1.

For NonInv $I a$ and $I b, 6$ parameters were fitted: 4 material parameters, the axial prestretch $\lambda_{z}$ and the outer radius in the unloaded configuration $\rho_{o}$. In Stålhand (2009) the fibre dispersion was not taken into account to reduce the number of fitting parameters. Hence, we set $\kappa$ to be 0 . When $\kappa$ was included, the problem seemed to be over-parameterised and resulted in poor estimates. The method provided by Schulze-Bauer (2003) was not able to include this additional parameter.

Non-invasive approach II (NonInv II) Here, we propose a new approach that does not consider the artery to be a thin-walled tube. This requires additional physiologically based assumptions. Similar to NonInv I, the force is assumed to be approximately constant in the physiological pressure range but no fixed value is set. The residual stresses related to an opening angle were not taken into account for NonInv II. The reason for this is discussed in section 4. Aside from the force condition, two more energy-based conditions were implemented. The first one is related to the energy across the arterial wall which is assumed to be approximately constant. This condition was derived from the reports that the principal stresses and strains are fairly uniform across the wall (Chuong and Fung, 1986; Rachev and Greenwald, 2003). The strain energy is uniquely defined by the principal strains, as can be seen in equation 2 . Hence, one can equally assume uniformity of the strain energy across the wall, which was used as a condition. The second condition states that at diastolic pressure, the amount of energy stored in the collagen fibres is close to the amount of energy stored in the matrix material. The validity of this assumption is discussed in section 4.

The aforementioned conditions yield the following objective function for NonInv II:

$$
\begin{aligned}
\varepsilon(\text { pars })= & \sum_{j=1}^{n}\left\{\left[w_{p}\left(P_{j}^{\text {mod }}-P_{j}^{F E M}\right)\right]^{2}\right. \\
& \left.+\left[w_{f}\left(\frac{F_{j}^{\text {mod }}}{A_{j}^{\text {mod }}}-\frac{F^{\text {average }}}{A_{j}^{\text {mod }}}\right)\right]^{2}\right\} \\
& +\sum_{k=1}^{m}\left\{\left[w_{\Psi 1}\left(\Psi_{\mathrm{k}}^{\text {dias }, \bmod }-\Psi^{\text {average }}\right)\right]^{2}\right. \\
& \left.+\left[w_{\Psi 2}\left(\Psi_{\mathrm{k}, \text { col }}^{\text {dias } \text { mod }}-\Psi_{\mathrm{k}, \operatorname{mat}}^{\text {dias } \bmod }\right)\right]^{2}\right\}
\end{aligned}
$$

$P_{j}^{m o d}$ and $F_{j}^{\text {mod }}$ are the pressures and forces calculated from the constitutive model for every $j$-th data record, as in (8) an (9). $P_{j}^{F E M}$ is the pressure used in the FE simulation and $F^{\text {average }}$ is calculated by using a polyfit function in Matlab and fitting a zero order polynomial to $\boldsymbol{F}^{\text {mod }}$. This second term ensures that the reduced axial force remains approximately constant in the physiological pressure range.

$\Psi_{\mathrm{k}}^{\text {dias,mod }}$ is the strain energy density at diastole across the wall thickness. $k$ represents different points throughout the wall going from 1 to $m, m$ being 11 in our case. $\Psi^{\text {average }}$ is calculated by using the polyfit function again, but now on $\boldsymbol{\Psi}^{\text {dias,mod }}$. This term minimizes the energy gradient across the wall at the diastolic level. The energy split in (2) enables us to separately monitor the contributions from collagen and matrix. The last part in (19) introduces the assumption that, at diastolic pressure, the energy contribution from collagen, $\boldsymbol{\Psi}_{\mathrm{k}, \mathrm{col}}^{\text {dias,mod }}$ is approximately the same as the energy contribution from the matrix material, $\Psi_{\mathrm{k}, \text { mat }}^{\text {dias, mod }}$. The index $k$ again represents values across the wall thickness. The weighting factors will again depend on the chosen units. They were set to 1 for $w_{p}, 10^{-2}$ for $w_{f}, 10^{-4}$ for $w_{\Psi 1}$ and $10^{-1}$ for $w_{\Psi 2}$. Since pressure is the only measurable variable, it has the highest importance in the parameter estimation scheme.

In NonInv II, 7 parameters were fitted, namely the 5 material parameters, as well as two geometrical parameters, the axial stretch $\lambda_{z}$ and the thickness in the unloaded configuration $H$. Note that in this approach we fit $\sin \alpha$ instead of $\alpha$ to have a more equally distributed search area for the parameters. The upper and lower boundary for $\alpha$ were changed accordingly.

\section{Results}

The parameters obtained from the different parameter identification approaches described in 2.4 are presented in Table 1. The first column in Table 1 gives the overview of all the parameters (material and geometrical) assigned in the FE model. The next two columns contain the results of the parameter fitting for the two invasive approaches. The next three columns give the parameter values obtained from the non-invasive approaches. Finally, the last column gives the root mean square error (RMSE) for each approach. The RMSE was calculated as follows:

$$
R M S E=\sqrt{\frac{\left(\boldsymbol{P}_{\text {mod }}-\boldsymbol{P}_{\boldsymbol{F E M}}\right)^{2}+\left(\frac{\boldsymbol{F}_{\text {mod }}}{\boldsymbol{A}_{\text {mod }}}-\frac{\boldsymbol{F}_{\boldsymbol{F} \text { EM }}}{\boldsymbol{A}_{\text {mod }}}\right)^{2}}{2 n}}
$$

Note that the different initial guesses used in the Multistart optimization process resulted in similar parameter 
Table 1 Parameters obtained from the different estimation approaches

\begin{tabular}{cccccccccc}
\hline \multicolumn{2}{c}{ Parameter } & $\begin{array}{c}\text { Fitting } \\
\text { range }\end{array}$ & $\begin{array}{c}\text { Scalining } \\
\text { factor }\end{array}$ & Actual & $\begin{array}{c}\text { Inv } \\
\text { noRS }\end{array}$ & $\begin{array}{c}\text { Inv } \\
R S\end{array}$ & $\begin{array}{c}\text { NonInv } \\
\text { Ia }\end{array}$ & $\begin{array}{c}\text { NonInv } \\
\text { Ib }\end{array}$ & $\begin{array}{c}\text { NonInv } \\
\text { II }\end{array}$ \\
\hline \multicolumn{1}{c}{} & & & & & & & & & \\
$\mu$ & {$[\mathrm{kPa}]$} & $0-1000$ & $10^{-3}$ & 15 & 14.51 & 14.98 & 15.53 & 7.61 & 19.2 \\
$k_{1}$ & {$[\mathrm{kPa}]$} & $0-1000$ & $10^{-3}$ & 80 & 65.14 & 79.91 & 41.99 & 28.43 & 58.3 \\
$k_{2}$ & {$[-]$} & $0-100$ & $10^{-3}$ & 5 & 4.14 & 4.99 & 3.18 & 2.81 & 3.8 \\
$\alpha$ & {$\left[{ }^{\circ}\right]$} & $0-90$ & $\pi / 180$ & 5 & 14.72 & 5.14 & 24.87 & 41.44 & 23.23 \\
$\kappa$ & {$[-]$} & $0-1 / 3$ & - & 0.2 & 0.1822 & 0.2 & $0^{*}$ & $0^{*}$ & 0.0841 \\
$\lambda_{z}$ & {$[-]$} & $1-2$ & - & 1.6 & $1.6^{*}$ & $1.6^{*}$ & 1.73 & 1.11 & 1.59 \\
$\Theta_{0}$ & {$\left[{ }^{\circ}\right]$} & $0-180$ & $\pi / 180$ & 120 & - & $120^{*}$ & - & - & - \\
$R_{\mathrm{O}}$ & {$[\mathrm{mm}]$} & $0.5-2$ & - & 1.14 & - & $1.14^{*}$ & - & - & - \\
$\rho_{\mathrm{O}}$ & {$[\mathrm{mm}]$} & $0.5-2$ & - & 0.78 & $0.78^{*}$ & - & 0.83 & 0.68 & - \\
$H$ & {$[\mathrm{~mm}]$} & $0.08-0.3$ & - & 0.14 & - & - & - & - & 0.13 \\
\hline $\mathrm{RMSE}$ & {$[\mathrm{kPa}]$} & - & - & - & 0.03 & 0.004 & 1.38 & 20.5 & 1.62 \\
\hline
\end{tabular}

* fixed value

sets. From the obtained solutions, the set with the lowest objective function value was chosen as the optimal parameter set.

From the results (Table 1 and Figure 4) it can be seen that in the approaches Inv noRS and Inv $R S$ both pressure and reduced axial force are fitted very well. The fitted parameters are very close to the actual parameters. When residual strains are disregarded, parameter $k_{1}$ is slightly lower, and parameter $\alpha$ is notably higher. In other words, ignoring the circumferential residual stress results in an underestimation of fibre stiffness and an overestimation of the main fibre angle.

NonInv I approach gives a reasonably good fit for both pressure and force but only if the parameter $\gamma$ is set to the correct value, which can not be the case in clinical applications. When this parameter is set to the value used in Schulze-Bauer and Holzapfel (2003) and Stålhand (2009) (NonInv Ib) the obtained parameters deviate from the real values and the reduced axial force is underestimated. The actual force is around $0.018 \mathrm{~N}$ whereas with NonInv Ib the force is slightly below 0.005 N. NonInv II provides considerably better results than NonInv Ib. The largest deviations from the ground truth were noticed with parameters $\alpha$ and $\kappa$ but they were still smaller than the errors in NonInv I. The average value for the reduced axial force from NonInv II was around $0.019 \mathrm{~N}$. In NonInv II the additional conditions are related to strain energy density (SED). The energy plots are given on Figure 5 for all the approaches. The energy is plotted for pressures from 0 to $200 \mathrm{mmHg}$. Note that, for the parameter estimation only physiological pressures were used (from 70 to $120 \mathrm{mmHg}$ ).

It is visible that in Inv $R S$ the parameters are not able to compensate for the disregarded circumferential residual stresses. This results in an overestimation of the strain energy. The error increases with in-

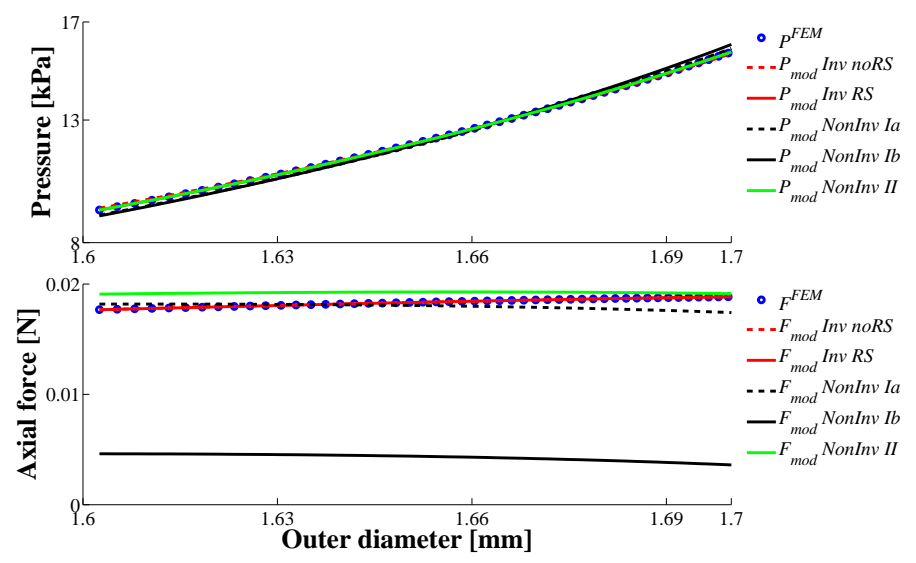

Fig. 4 Pressure-outer radius curves plotted with parameters from: the FEM (circles), the invasive approach without residual strains (red dashed lines), the invasive approach with residual strains (red full line), and the non-invasive approach Ia with $\gamma=0.8565$ (black dashed lines), the non-invasive approach Ib with $\gamma=0.59$ (black full line) and the new noninvasive approach (green full line).

creasing pressure. Since both non-invasive approaches do not take residual stresses in consideration, the energy overestimation can also be seen on the other three energy plots. The NonInv Ia (Figure $5 \mathrm{~b}$ ) gives an approximately equally good energy prediction as NonInv II (Figure 5d), but the latter one does not need knowledge about the stress ratio $\gamma$. If $\gamma$ is unknown and set to a wrong value (NonInv $I b$ ), the energy overestimation becomes more severe (Figure $5 \mathrm{c}$ ). Sensitivity of the approach NonInv I to parameter $\gamma$ is displayed on Figure 6 . From the figure it can be concluded that an under- or overestimation of $\gamma$ has a large effect on the final error. 


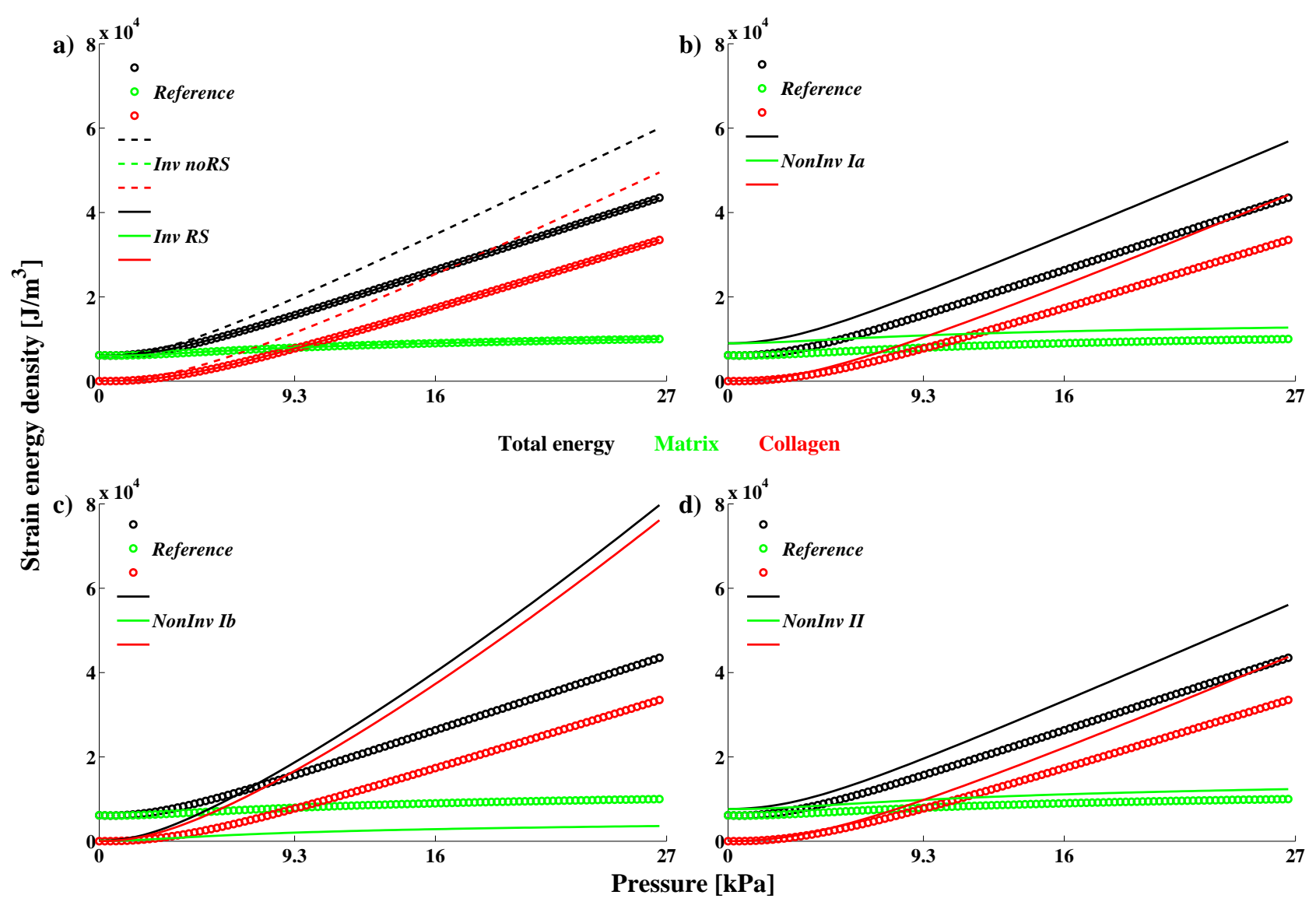

Fig. 5 The total SED (black), the amount of the SED from the matrix material (green) and collagen (red). The SED for the different approaches is always plotted against the reference (energy calculated with the actual parameters). On a) both of the invasive approaches are plotted, b) Non-invasive approach Ia with $\gamma=0.8565$, c) Non-invasive approach Ib with $\gamma=0.59$, d) Non-invasive approach II. Pressure of $9.3 \mathrm{kPa}$ corresponds to diastole and $16 \mathrm{kPa}$ to systole.

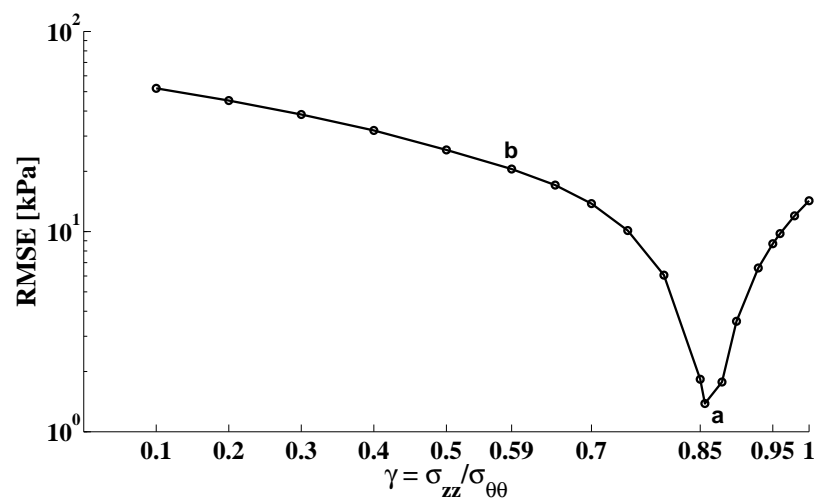

Fig. 6 Sensitivity of the approach NonInv I on the parameter $\gamma$ (ratio of axial and circumferential stress at the pressure of $13.3 \mathrm{kPa})$. a) Value used in NonInv Ia; b) value used in NonInv Ib.

\section{Discussion}

In this paper finite element modelling was used to evaluate different parameter fitting approaches to fit constitutive arterial models to data that can be achieved in various in vivo and ex vivo experiments. This evaluation method is not a replacement for an experimental validation where results of the non-invasive parameter estimation are compared with the results from in vitro mechanical experiments, such as inflation-extension or planar biaxial tests. On the other hand, it does offer an objective method to quantitatively evaluate these parameter identification approaches, without being influenced by typical experiment-related errors.

Here, we make use of a virtual, controlled and known environment where a FE model of the aorta with a fixed geometry and known material parameters serves as a ground truth for the comparison of the evaluated methods. In this way, purely the parameter estimation methods are evaluated, while the interference of various measurement uncertainties and artefacts, difficulties related to the measurement of opening angles and the thickness of the aortic wall are excluded from the analysis.

A possible limitation of this study is the assumption of a perfectly cylindrical segment of the aorta. The 
consequences of a real aortic geometry on the parameter identification are unknown and additional analysis of its effects is necessary. Alternatively, inverse finite element based fitting can be used to take the actual geometry into account (Wittek et al., 2013). The limiting factor of this approach is the high computational cost imposed by iterative solving of the FE problem. However, it was demonstrated in Famaey et al. (2013b) that by application of dedicated hardware the solution times can be greatly decreased. Future improvements in this respect might allow fast computation of the parameters with a more realistic geometrical representation. Note that the inverse $\mathrm{FE}$ approach also requires $3 \mathrm{D}$ image information, making it also more tedious than a relatively straightforward, pressure-diameter measurement.

The second contribution of this study is a new energybased method for non-invasive material property estimation of arterial tissue. The method incorporates extra conditions related to the physiological behaviour of the tissue. A multi-objective minimisation is performed, allowing the solution to violate a certain condition at a cost given by the weight factor. One condition states that the energy contribution of the matrix material is close to the collagen energy contribution at diastolic pressure. This condition was derived from studies performed on animals and humans. Roach and Burton (1957) reported that collagen gets recruited around diastolic pressure in humans, while elastin is the load bearing unit at lower pressures. Wolinsky and Glagov (1964) reported similar findings on rabbit aortas. They report that at and above physiological pressures collagen fibres bear most of the loading. We noticed similar

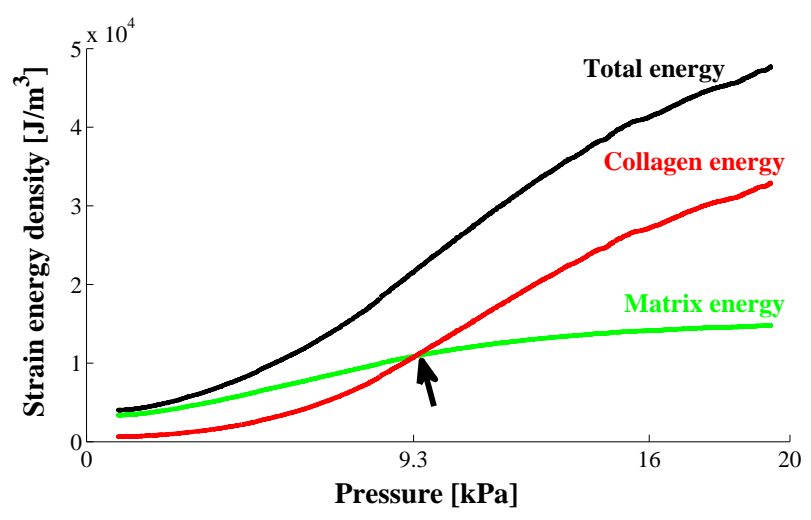

Fig. 7 Total energy and a split of it to a matrix and collagen contribution for one rat. The arrow marks the crossing pressure where the collagen contributions becomes higher than the matrix contribution.

behaviour in rats. From the inflation-extension experiments performed on six rat abdominal aortas (Famaey et al., 2012), the invasive approach (Inv noRS) was used to obtain the parameters. The total SED was calculated as well as the contributions from collagen and matrix material. Figure 7 shows the obtained energy curves. It was observed that elastin contributes more below diastolic pressure while above it, collagen becomes more important. Pressures at which this crossing occurs, called 'crossing pressures' (marked with the arrow on Figure 7), were ranging from 61 to $83 \mathrm{mmHg}$ with an average of $70.5 \mathrm{mmHg}$.

Another condition used in our new fitting approach is the minimization of the energy gradient across the arterial wall. In literature, the principal stresses and strains are reported to be moderately constant across the wall (Chuong and Fung, 1986; Fung et al., 1979; Rachev and Greenwald, 2003). Commonly, the circumferential stress gradient is minimized across the wall. By minimizing the energy gradient, the stress gradients in all three directions are indirectly minimized. Figure 8 displays stresses and strain energy density plots across the wall at diastolic pressure. This was done using the actual parameters as well as with the optimized parameter values from the two invasive parameter identification approaches (Inv noRS and Inv $R S$ ) and the newly proposed non-invasive parameter estimation approach (NonInv II). Note that the two other non-invasive approaches (NonInv Ia and NonInv Ib) are not included in these plots, since they consider an artery to be a thin-walled tube and hence fail to capture transmural effects. As can be seen in the figure, the strain energy density stays approximately constant across the wall in the reference case. The NonInv II approach does not take into account residual stresses so it is not able to fully capture this effect. However, thanks to the imposed condition, it does already perform slightly better than the Inv noRS approach.

One limitation of the proposed approach arises from the fact that the method was tested based on one set of parameters derived from experiments on rat aortas. Though we would expect to obtain the same qualitative results for, e.g., human arterial tissue if the same constitutive model is used, this needs to be proven. However, to be able to perform a reliable FE simulation representing the in vivo cyclic pressurization (as done in this study), quite a number of parameters need to be known. Apart from the correct geometry and the actual material parameters, a realistic opening angle and in vivo prestretch value should be used. The authors have not found any publications where the combination of all these parameters is reported. Using wrong or incomplete parameters would imply that the reduced axial force will not be independent from the pressure (i.e. fairly constant over the physiological pressure range), which is one of the used assumptions. 

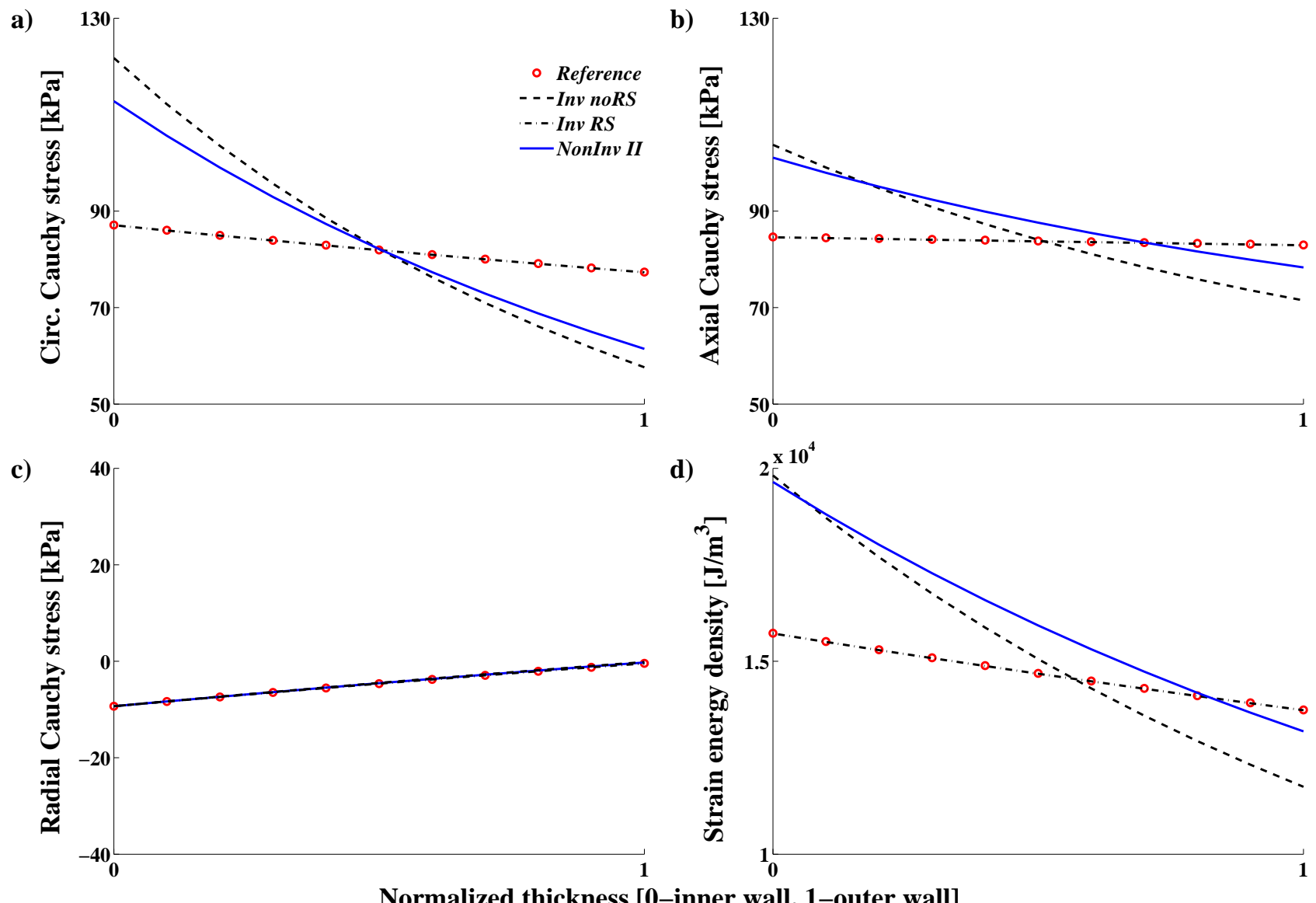

Normalized thickness [0-inner wall, 1-outer wall]

Fig. 8 Stresses and strain energy density at diastolic pressure $(9.3 \mathrm{kPa})$ across the arterial wall ; a) circumferential Cauchy stress; b) axial Cauchy stress; c) radial Cauchy stress; d) strain energy density. On each graph, the reference situation is plotted as well as the values obtained from the two invasive approaches (Inv noRS and Inv $R S$ ) and the newly proposed non-invasive parameter identification approach (NonInv II).

Secondly, the constitutive model used here was developed for healthy tissue and does not incorporate the active contribution of the smooth muscle cells. In clinical practice, the patients are expected to have some form of cardiovascular disease and it is well known that the active contribution of the wall plays an important role (Böl et al., 2012; Murtada et al., 2010; Famaey et al., 2013a). The used model also consists of a single layer while in reality arteries have three distinct layers. However, more complex models taking into account the pathology, more accurate description of the structure of the wall or the active contribution would lead to an increased number of parameters and an overparameterisation. More conditions would have to be added to overcome this issue. It is worth noting that the technique of adding more conditions will on the one hand introduce dependencies between the parameters which can reduce the over-parameterisation. On the other hand it could make the minimisation harder or even impossible if the model does not allow for the included conditions, as discussed in Stålhand and Klarbring (2005).

At the moment, the circumferential residual stresses are not included in the new fitting approach. In van der Horst et al. (2012) a set of in vitro experiments on porcine and human coronary artery segments was performed to evaluate the collagen fibre orientation. They introduced residual stresses in their optimization scheme by means of an objective function which ensures that the circumferential stress gradient is minimal at physiological pressure $13.3 \mathrm{kPa}$. They performed the optimization with and without this assumption and concluded that the effect of including the opening angle on the parameters was very small. In our study, the effect of the residual stresses was investigated by comparing the results from Inv noRS and Inv RS. From Table 1 it can be seen that disregarding the circumferential residual stress resulted in an underestimation of collagen fibre stiffness, $k_{1}$ and an overestimation of the fibre angle, $\alpha$. However, including the opening angle, i.e. by 
introducing an additional fitting parameter, resulted in over-parameterisation, making it impossible to obtain a unique set of parameters. Our decision not to include the opening angle was also based on the results of a sensitivity analysis performed on all parameters listed in Table 1 which showed that the opening angle was the least sensitive of these.

From the results in Table 1 and the SED plots on Figure 5 it is visible that NonInv Ia and NonInv II give approximately equally good results. However, the used value for the stress ratio $\gamma$ was acquired from the FE simulation. In reality, it is impossible to know this ratio specifically for each patient. This is a major disadvantage of the method NonInv I. To emphasize this, an estimation of the parameters was performed using the correct ratio (known from the simulation) and the incorrect ratio, i.e. the one used in Schulze-Bauer and Holzapfel (2003) and Stålhand (2009), which was based on experimental in vitro data. The method only performs well when the correct ratio is used (Table 1). The same conclusion can be drawn from the results of the sensitivity analysis of $\gamma$ shown on Figure 6. This makes the method NonInv II more 'clinically-friendly'. In addition, NonInv I considers the artery to be a thinwalled tube, which is not the case for NonInv II.

However, even NonInv II under- and overestimated some parameters. The largest errors were related to the parameter $\alpha$, i.e. a parameter related to the contribution of collagen fibres describing the anisotropy between the axial and the circumferential direction. $\alpha$ was overestimated with both non-invasive methods. This can be partially explained by the fact that the circumferential residual stresses were not taken into account. This statement is supported by the results from the two invasive approaches. It can be seen in Table 1 that if the residual stresses are assumed to be zero, the fitted value for $\alpha$ is close to $14.72^{\circ}$, which was an overestimation of almost $200 \%$. Another explanation can be that $\alpha$, when fitted, loses its physical meaning. Similar remarks were reported in Haskett et al. (2010) where they compared results for $\alpha$ obtained by small-angle light scattering (SALS) and parameter fitting from biaxial experiments performed on human aortic samples. For a group of samples which had an average measured angle from SALS of $14.98^{\circ}$, they obtained a result of $48.7^{\circ}$ from the fitting. The reported conclusion was that the regression fit was unable to predict the true angle of preferred fibre alignment.

The stiffness of the collagen fibres $\left(k_{1}\right)$ is underestimated if the NonInv II approach is used. The same remarks as for $\alpha$, related to the opening angle, are valid here. The underestimation comes as a result of not taking into account the opening angle. If the unloaded con- figuration is chosen as a reference configuration, it is assumed that in the beginning collagen fibres are not contributing at all, while in reality they are already slightly recruited.

In summary, all non-invasive approaches performed in this paper give results that deviate from the real values. Hence, the parameters obtained as such should be interpreted and used with great caution. It can be expected that if the deviation in this idealized case is already present, the results from actual non-invasive measurements and parameter estimation will vary even more. The obtained parameters, if used for simulation purposes, will result in an overestimation of the strain energy and consequently an overestimation of the stresses.

Nevertheless, the NonInv II approach already enables us to study relative patient variations in material properties based on non-invasive population studies such as Asklepios study (Rietzschel et al., 2007). This will already bring us one step closer to clinical use.

To conclude, this study suggests a new energy-based method for the constitutive parameter estimation of arterial tissue from non-invasive pressure and diameter measurements. The method is more accurate than the previous method due to the thick-walled tube approach and the enforcement of two energy-related conditions. Additionally, it does not require any knowledge regarding the ratio of stresses making it also more robust and more appropriate for clinical use.

Acknowledgements This research was supported by Research Foundation Flanders (FWO Vlaanderen). 


\section{APPENDIX}

\section{List of symbols}

$\Psi \quad$ strain energy density function

$\Psi_{\text {mat }} \quad$ isotropic contribution of the extracellular matrix material to the strain energy density function

$\Psi_{\text {col }} \quad$ anisotropic contribution of the collagen fibre families to the strain energy density function

$I_{1} \quad$ first invariant of the right Cauchy-Green strain tensor

$I_{4} \quad$ invariant related to the orientation of the collagen fibre families

$\lambda_{i} \quad i=r, \theta, z$, three principal stretch ratios in the radial, circumferential and the axial directions of the artery, respectively

$\mu \quad$ stress-like parameter representing the stiffness of the matrix material

$k_{1} \quad$ stress-like parameter representing the stiffness of the collagen fibres

$k_{2} \quad$ dimensionless parameter of the collagen fibres

$\alpha \quad$ angle between the mean collagen fibre direction and the circumferential direction of the artery

$\kappa \quad$ parameter related to the dispersion of the collagen fibres

$L \quad$ initial axial sample length

$l \quad$ deformed axial sample length

$R_{\mathrm{O}} \quad$ outer radius in the stress-free configuration

$R_{\mathrm{i}} \quad$ inner radius in the stress-free configuration

$\rho_{\mathrm{o}} \quad$ outer radius in the unloaded configuration

$\rho_{\mathrm{i}} \quad$ inner radius in the unloaded configuration

$r_{\mathrm{o}} \quad$ outer radius in the loaded configuration

$r_{\mathrm{i}} \quad$ inner radius in the loaded configuration

$R \quad$ initial radius at a certain position

$r \quad$ deformed radius at a certain position

$\Theta_{0} \quad$ opening angle in the stress-free configuration

$\boldsymbol{F}_{1} \quad$ deformation gradient relating the stress-free and the loaded configuration

$\boldsymbol{F}_{\mathbf{2}}$ deformation gradient relating the unloaded and the loaded configuration

$\mathcal{R}_{0} \quad$ stress-free configuration

$\mathcal{R}_{1} \quad$ unloaded configuration

$\mathcal{R}_{2} \quad$ loaded configuration

$R, \theta, z \quad$ radial, circumferential and axial cylindrical coordinates in the stress-free configuration

$\rho, \phi, \xi$ radial, circumferential and axial cylindrical coordinates in the unloaded configuration

$r, \theta, z \quad$ radial, circumferential and axial cylindrical coordinates in the stress-free configuration

$\Lambda$ axial stretch ratio between the stress-free and the unloaded configuration

$\lambda \quad$ axial stretch ratio between the unloaded and the loaded configuration

$\lambda_{z} \quad$ total stretch in the axial direction

$H \quad$ wall thickness in the stress-free and unloaded configuration

$h \quad$ wall thickness in the loaded configuration

$j \quad$ index going from 1 to $n, n$ being the total number of data points considered

$k \quad$ index representing different points throughout the arterial wall, going from 1 at the inner wall to $m$ at the outer wall

\begin{tabular}{|c|c|}
\hline $\boldsymbol{F}^{F E M}$ & $\begin{array}{l}\text { reduced axial forces extracted from the } \\
\text { simulation }\end{array}$ \\
\hline & outer radii extracted from the simulation \\
\hline $\boldsymbol{P}^{F E M}$ & pressures extracted from the simulation \\
\hline pars & vector of fitted parameters \\
\hline$w_{p}$ & $\begin{array}{l}\text { weighting factor for the pressure in the } \\
\text { objective function }\end{array}$ \\
\hline$w_{f}$ & $\begin{array}{l}\text { weighting factor for the force in the } \\
\text { objective function }\end{array}$ \\
\hline $\boldsymbol{P}^{\text {mod }}$ & $\begin{array}{l}\text { intraluminal pressures predicted by the } \\
\text { function } \Psi\end{array}$ \\
\hline $\boldsymbol{F}^{\bmod }$ & $\begin{array}{l}\text { reduced axial forces predicted by the } \\
\text { function } \Psi\end{array}$ \\
\hline $\boldsymbol{A}^{\bmod }$ & $\begin{array}{l}\text { cross sectional area predicted by the } \\
\text { function } \Psi\end{array}$ \\
\hline$\lambda_{\mathrm{o}}$ & circumferential axial stretch at the outer wall \\
\hline$\lambda_{\mathrm{i}}$ & circumferential axial stretch at the inner wall \\
\hline $\boldsymbol{\sigma}_{\theta \theta}^{\bmod }$ & $\begin{array}{l}\text { circumferential stresses predicted by the } \\
\text { function } \Psi\end{array}$ \\
\hline$\sigma_{\theta \theta}$ & $\begin{array}{l}\text { circumferential stresses calculated by } \\
\text { enforcing the equilibrium } \\
\text { function } \Psi\end{array}$ \\
\hline$\sigma_{\boldsymbol{z} z} \bmod$ & axial stresses predicted by the function $\Psi$ \\
\hline$\sigma_{z z}$ & $\begin{array}{l}\text { axial stresses calculated by enforcing } \\
\text { the equilibrium }\end{array}$ \\
\hline$F^{e s t}$ & estimated reduced axial force \\
\hline $\bar{P}$ & an arbitrary pressure, e.g. $100 \mathrm{mmHg}$ \\
\hline $\bar{r}_{\mathrm{i}}$ & inner radius related to the pressure $\bar{P}$ \\
\hline $\bar{h}_{\mathrm{i}}$ & wall thickness related to the pressure $\bar{P}$ \\
\hline$\gamma$ & $\begin{array}{l}\text { ratio of the axial to the circumferential } \\
\text { stresses at the pressure } \bar{P}\end{array}$ \\
\hline$w_{\Psi 1}$ & $\begin{array}{l}\text { weighting factor for the strain energy density } \\
\text { across the wall thickness }\end{array}$ \\
\hline$w_{\Psi 2}$ & $\begin{array}{l}\text { weighting factor for the strain energy density } \\
\text { at diastole }\end{array}$ \\
\hline Faverage & average of $\boldsymbol{F}^{\text {mod }}$ \\
\hline $\begin{array}{l}\Psi^{\text {dias }, \bmod } \\
\Psi_{\text {average }}\end{array}$ & $\begin{array}{l}\text { strain energy density at diastole } \\
\text { average of } \Psi_{\text {dias }} \text { mod }\end{array}$ \\
\hline$\Psi_{\text {dias, } \bmod }$ & collagen strain energy de \\
\hline \begin{tabular}{|}
$\Psi_{\text {mat }}^{\text {coll }}$ dias mod \\
mat
\end{tabular} & matrix strain energy density at diastole \\
\hline
\end{tabular}

\section{References}

Böl, M., Abilez, O. J., Assar, A. N., Zarins, C. K., and Kuhl, E. (2012). In vitro/in silico characterization of active and passive stresses in cardiac muscle. International Journal for Multiscale Computational Engineering, 10(2):171-188.

Cardamone, L., Valentín, A., Eberth, J. F., and Humphrey, J. D. (2009). Origin of axial prestretch and residual stress in arteries. Biomech Model Mechanobiol, 8(6):431-446.

Chuong, C. J. and Fung, Y. C. (1986). Residual stress in arteries. In Schmid-Schönbein, G. W., Woo, S. L.Y., and Zweifach, B. W., editors, Frontiers in Biomechanics, Part II, pages 117-129. Springer New York.

Famaey, N., Sommer, G., Vander Sloten, J., and Holzapfel, G. A. (2012). Arterial clamping: finite element simulation and in vivo validation. $J$ Mech 
Behav Biomed Mater, 12:107-118.

Famaey, N., Vander Sloten, J., and Kuhl, E. (2013a). A three-constituent damage model for arterial clamping in computer-assisted surgery. Biomech Model Mechanobiol, 12(1):123-136.

Famaey, N., Štrbac, V., and Vander Sloten, J. (2013b). Intraoperative Damage Monitoring of Endoclamp Balloon Expansion Using Real-Time Finite Element Modeling. Springer.

Fung, Y. C., Fronek, K., and Patitucci, P. (1979). Pseudoelasticity of arteries and the choice of its mathematical expression. Am J Physiol, 237(5):H620H631.

Gasser, T. C., Ogden, R. W., and Holzapfel, G. A. (2006). Hyperelastic modelling of arterial layers with distributed collagen fibre orientations. $J R$ Soc Interface, 3(6):15-35.

Haskett, D., Johnson, G., Zhou, A., Utzinger, U., and Vande Geest, J. (2010). Microstructural and biomechanical alterations of the human aorta as a function of age and location. Biomech Model Mechanobiol, $9(6): 725-736$.

Holzapfel, G. A., Gasser, T. C., and Ogden, R. W. (2000). A new constitutive framework for arterial wall mechanics and a comparative study of material models. Journal of elasticity and the physical science of solids, 61, Issue 1-3:1-48.

Humphrey, J. D. (2002). Cardiovascular solid mechanics: Cells, tissues and organs. Springer.

Masson, I., Boutouyrie, P., Laurent, S., Humphrey, J. D., and Zidi, M. (2008). Characterization of arterial wall mechanical behavior and stresses from human clinical data. J Biomech, 41(12):2618-2627.

Murtada, S.-I., Kroon, M., and Holzapfel, G. A. (2010). A calcium-driven mechanochemical model for prediction of force generation in smooth muscle. Biomech Model Mechanobiol, 9(6):749-762.

Nichols, M., Townsend, N., Scarborough, P., and Rayner, M. (2013). European cardiovascular disease statistics 4th edition 2012: Euroheart ii. Eur Heart J, 34(39):3007.

Ogden, R. W. (2009). Anisotropy and nonlinear elasticity in arterial wall mechanics. In Holzapfel, G. A. and Ogden, R. W., editors, Biomechanical Modelling at the Molecular, Cellular and Tissue Levels, pages 179-258, Wien, New York. Springer-Verlag. CISM Courses and Lectures no. 508.

Rachev, A. and Greenwald, S. E. (2003). Residual strains in conduit arteries. J Biomech, 36(5):661670.

Rietzschel, E.-R., De Buyzere, M. L., Bekaert, S., Segers, P., De Bacquer, D., Cooman, L., Van Damme, P., Cassiman, P., Langlois, M., van Oostveldt, P.,
Verdonck, P., De Backer, G., Gillebert, T. C., and , A. I. (2007). Rationale, design, methods and baseline characteristics of the asklepios study. Eur J Cardiovasc Prev Rehabil, 14(2):179-191.

Roach, M. R. and Burton, A. C. (1957). The reason for the shape of the distensibility curves of arteries. Can J Biochem Physiol, 35(8):681-690.

Schulze-Bauer, C. A. J. and Holzapfel, G. A. (2003). Determination of constitutive equations for human arteries from clinical data. J Biomech, 36(2):165169.

Stålhand, J. (2009). Determination of human arterial wall parameters from clinical data. Biomech Model Mechanobiol, 8(2):141-148.

Stålhand, J. and Klarbring, A. (2005). Aorta in vivo parameter identification using an axial force constraint. Biomech Model Mechanobiol, 3(4):191-199.

van der Horst, A., van den Broek, C. N., van de Vosse, F. N., and Rutten, M. C. M. (2012). The fiber orientation in the coronary arterial wall at physiological loading evaluated with a two-fiber constitutive model. Biomech Model Mechanobiol, 11(3-4):533542 .

Vito, R. P. and Dixon, S. A. (2003). Blood vessel constitutive models-1995-2002. Annu Rev Biomed Eng, 5:413-439.

Weizsäcker, H. W., Lambert, H., and Pascale, K. (1983). Analysis of the passive mechanical properties of rat carotid arteries. J Biomech, 16(9):703-715.

Wittek, A., Karatolios, K., Bihari, P., Schmitz-Rixen, T., Moosdorf, R., Vogt, S., and Blase, C. (2013). In vivo determination of elastic properties of the human aorta based on 4d ultrasound data. J Mech Behav Biomed Mater, 27:167-183.

Wolinsky, H. and Glagov, S. (1964). Structural basis for the static mechanical properties of the aortic media. Circ Res, 14:400-413. 